\title{
Policondrite Recidivante na Infância: Relato de Caso e Revisão da Literatura
}

\section{Relapsing Polychandritis in the Childhood: Case Report and Literature Review}

\author{
André Cavalcanti( ${ }^{(1)}$, Cássia Maria P. L. Barbosa ${ }^{(2)}$, Claudio Arnaldo Len ${ }^{(3)}$, \\ Maria Teresa R. A. Terreri ${ }^{(4)}$, Maria Odete E. Hilário(5)
}

\section{RESUMO}

A policondrite recidivante é uma doença rara caracterizada por episódios recorrentes de inflamação das cartilagens articular, auricular, nasal e laringotraqueal de intensidade variável. O diagnóstico diferencial inclui as doenças difusas do tecido conjuntivo, as vasculites e as síndromes febris periódicas na infância. Relatamos o caso de um adolescente de 11 anos com artrite de joelhos, conjuntivite aguda, acometimento de orelha, nariz e vias aéreas superiores cuja doença foi controlada com o uso de corticosteróides e imunossupressores. Os achados clínicos e laboratoriais são discutidos e comparados com os dados da literatura.

Palavras-chave: policondrite recidivante, artrite, inflamação, cartilagem, nariz, condrite auricular.

\section{INTRODUÇÃO}

A policondrite recidivante (PR) é uma doença multissistêmica potencialmente grave, de origem auto-imune, caracterizada por inflamação e destruição das cartilagens auricular, nasal, laringotraqueal e articular. Eventualmente, podem ser acometidos outros tecidos ricos em proteoglicanos, como olhos, vasos sangüíneos, coração e rins.

É uma doença rara, incomum na infância e na adolescência, sendo sua incidência anual em Rochester, Minnesota, estimada em 3,5 casos por milhão de habitantes, com pico

\begin{abstract}
Relapsing polychondritis is a rare disease characterized by recurrent episodes of inflammation of the articular, auricular, nasal and laryngotracheal cartilages of variable intensity. Differential diagnosis includes connective tissue diseases, vasculitis and periodic febrile syndromes of childhood. We report a case of 11 year-old boy with knee arthritis, acute conjunctivitis, ear, nose and upper airway cartilage involvement whose disease was controlled with the use of corticosteroids and immunosuppressive drugs. The clinical and laboratory findings were discussed and compared with case reports from literature.
\end{abstract}

Keywords: relapsing polychondritis, arthritis, inflammation, cartilage, nose, auricular chondritis.

entre a quarta e quinta décadas de vida. Sua apresentação na infância ou na adolescência é incomum ${ }^{(1,2)}$. Alguns achados clínicos como condrites auricular e nasal são altamente sugestivos de PR; o diagnóstico diferencial é restrito e inclui as doenças difusas do tecido conjuntivo, as vasculites, como a granulomatose de Wegener e a poliarterite nodosa, as doenças auto-inflamatórias (síndromes febris periódicas), as doenças granulomatosas, como a sarcoidose e a tuberculose e algumas doenças infecciosas com manifestações cutâneas (sífilis e hanseníase).

Recebido em 18/01/07. Aprovado, após revisão, em 11/05/07.

Disciplina de Alergia, Imunologia e Reumatologia do Departamento de Pediatria da Universidade Federal de São Paulo - Escola Paulista de Medicina (Unifesp/EPM).

1. Residente da Disciplina de Alergia, Imunologia e Reumatologia do Departamento de Pediatria da Unifesp/EPM.

2. Médica assistente da Disciplina de Alergia, Imunologia e Reumatologia do Departamento de Pediatria da Unifesp/EPM

3. Professor adjunto da Disciplina de Alergia, Imunologia e Reumatologia do Departamento de Pediatria da Unifesp/EPM

4. Professora afiliada da Disciplina de Alergia, Imunologia e Reumatologia do Departamento de Pediatria da Unifesp/EPM

5. Professora associada e responsável pelo Setor de Reumatologia da Disciplina de Alergia, Imunologia e Reumatologia do Departamento de Pediatria Unifesp/EPM. Endereço para correspondência: Prof. Dr. Claudio Arnaldo Len, Rua dos Otonis, 725, CEP 04025-002, São Paulo, SP, Brasil, Telefax: (11) 5579-1590, e-mail: len.cl@terra.com.br. 
A seguir, relatamos e discutimos o caso de um adolescente de 11 anos com quadro clínico compatível com o da PR, internado na enfermaria de Pediatria do Hospital São Paulo/Unifesp.

\section{RELATO DE CASO}

Menino de 11 anos, etnia negra, previamente hígido, apresentou hiperemia no olho esquerdo há seis meses. Procurou oftalmologista que diagnosticou conjuntivite e prescreveu medicação tópica sem melhora. Evoluiu com febre de $38,5^{\circ} \mathrm{C}$, perda de peso $(8 \mathrm{~kg})$ e aumento de volume e dor no joelho esquerdo. Foi internado em um hospital de atendimento secundário na Cidade de São Paulo. Exames laboratoriais na admissão: Hemoglobina (Hb) 10,9 g/dl, 6.900 glóbulos brancos $/ \mathrm{mm}^{3}$ (diferencial normal), 436.000 plaquetas $/ \mathrm{mm}^{3}$ e velocidade de hemossedimentação (VHS) de $117 \mathrm{~mm}$ na primeira hora. Foi feita uma hipótese diagnóstica de artrite séptica no joelho esquerdo, sendo realizada limpeza cirúrgica com biópsia de sinóvia e prescritos antibióticos de amplo espectro. A biópsia mostrou infiltrado inflamatório misto, proliferação vascular e depósitos de fibrina. Nesse período, apresentou artrite aditiva em joelho direito, tornozelos e articulação interfalangeana proximal do quinto quirodáctilo da mão esquerda (Figura 1). Concomitantemente, apresentou dor, edema e hiperemia em pavilhão auricular direito, sem acometimento do lóbulo da orelha (Figura 2). Devido à persistência do quadro clínico após dois meses de internação sem esclarecimento de diagnóstico, o paciente foi encaminhado para o Hospital São Paulo.

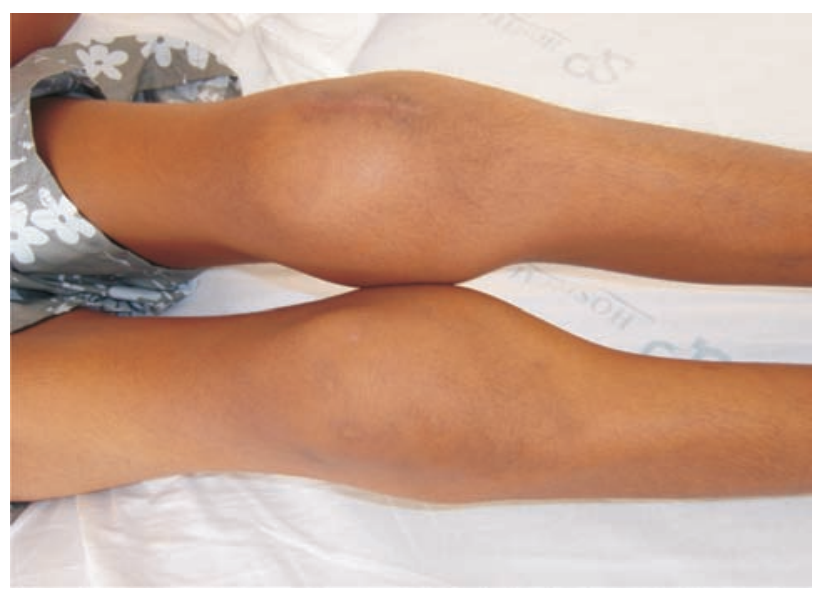

Figura 1 - Artrite deformante em joelhos.

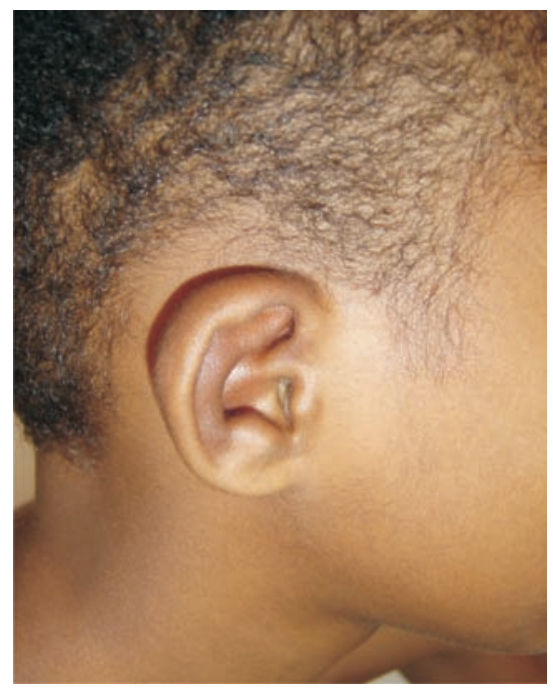

Figura 2 - Condrite em orelha direita.

Ao exame de admissão, além da artrite, foram observados queda do estado geral, febre alta $\left(39^{\circ} \mathrm{C}\right)$, achatamento do nariz (Figura 3), dor à palpação de articulação sacroilíaca direita e entesite em calcâneos. Negava quadro infeccioso pregresso e atividade sexual antes do aparecimento das manifestações articulares. Foram realizados outros exames: anticorpo antinúcleo, fator reumatóide, HLA-B27 e anticorpo contra citoplasma de neutrófilos negativos. Realizados ecocardiograma, radiografia de tórax e urina tipo I que foram normais. PPD não reagente. Feita hipótese diagnóstica de PR (poliartrite não-erosiva soronegativa com condrites nasal e auricular e inflamação ocular), foram iniciados naproxeno (15 mg/kg/dia), pulsoterapia com metilprednisolona ( $1 \mathrm{~g}$ por via endovenosa, por três dias), seguidos de prednisona $60 \mathrm{mg} /$ dia e ciclosporina na dose de $3 \mathrm{mg} / \mathrm{kg} /$ dia com aumento progressivo para $5 \mathrm{mg} / \mathrm{kg} /$ dia. Foi observada significativa melhora do estado geral, da poliartrite e dos processos inflamatórios auricular e nasal. Depois de 40 dias de internação, o paciente recebeu alta e foi encaminhado para o ambulatório de reumatologia pediátrica.

Tendo em vista a persistência da artrite moderada em ambos joelhos por seis meses após a alta, foi optado pela associação do metotrexato na dose de $25 \mathrm{mg}$ /semana por via oral. A biomicroscopia para pesquisa de uveíte crônica foi normal.

Após um ano de evolução da doença e em uso de ciclosporina $200 \mathrm{mg}$ /dia, metotrexato $25 \mathrm{mg} / \mathrm{sem}$ e prednisona $5 \mathrm{mg} /$ dia, o paciente apresentou um quadro insidioso de tosse e estridor respiratório alto, com piora progressiva da respiração, que resultou em intubação endotraqueal e 


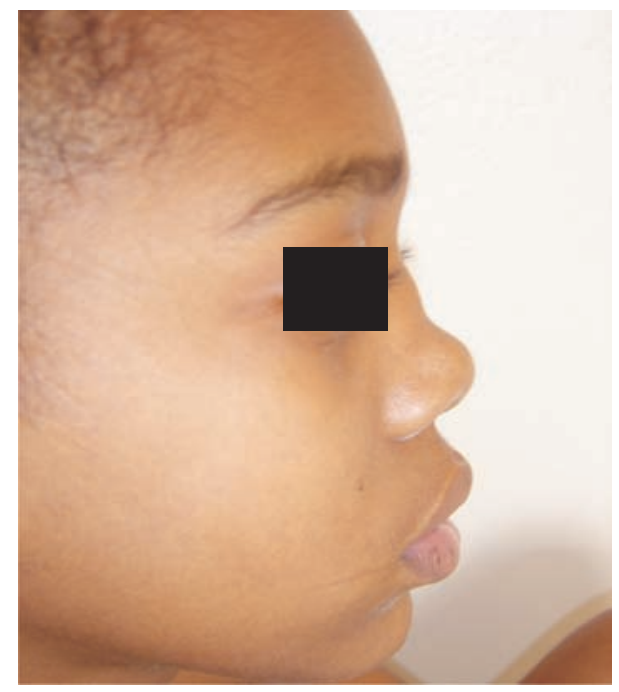

Figura 3 - Condrite nasal com deformidade tipo nariz em sela.

posterior transferência para unidade de terapia intensiva pediátrica. Na ocasião, o paciente apresentou VHS de 100 $\mathrm{mm}$ na primeira hora. Foi instituída novamente pulsoterapia com metilprednisolona ( $1 \mathrm{~g}, \mathrm{EV}$, por três dias). No terceiro dia, foi realizada pulsoterapia com ciclofosfamida $500 \mathrm{mg} / \mathrm{m}^{2}$. Apresentou melhora progressiva dos parâmetros ventilatórios e, 72 horas após, foi extubado. Teve alta depois de uma semana, sem sintomas respiratórios, sendo mantidos o metotrexato e a ciclosporina. Nessa internação, foi realizada tomografia computadorizada de alta resolução da árvore traqueobrônquica que mostrou espessamento da mucosa respiratória no nível da laringe.

No momento, o paciente continua em seguimento ambulatorial e desde a alta não apresentou novas recorrências. Mantém suas atividades diárias com limitação parcial às atividades físicas.

\section{DISCUSSÃO}

A raridade da $\mathrm{PR}$ na faixa etária pediátrica despertou nosso interesse para a descrição deste caso. Os comprometimentos sistêmico e da cartilagem das vias aéreas superiores foram determinantes da gravidade do quadro clínico deste paciente. No entanto, a instituição precoce do tratamento possibilitou a pronta recuperação, mas não foi capaz de impedir a recorrência do processo inflamatório, característico dessa doença ${ }^{(3)}$.

A etiologia da PR é desconhecida. Algumas evidências sugerem patogênese auto-imune, tais como: presença de auto-anticorpos contra o colágeno tipo II, detecção de imu- nidade celular contra constituintes da cartilagem, associação com HLA-DR4 e com outras doenças auto-imunes ${ }^{(1,4-6)}$. Animais imunizados com colágeno tipo II apresentaram doença semelhante à PR do adulto com condrite auricular e inflamação $\operatorname{articular}^{(1,7)}$. A alteração inicial em cartilagens afetadas pela PR é a perda da basofilia normal que corresponde à perda de proteoglicanos, seguida pela presença de processo inflamatório e diminuição do número de condrócitos nas áreas de destruição da cartilagem e, por fim, a cartilagem é substituída por tecido de granulação ${ }^{(8,9)}$.

O diagnóstico da PR é clínico e, desde 1976, os critérios de McAdam são utilizados na prática diária ${ }^{(10)}$ (Quadro 1). Os critérios preenchidos pelo nosso paciente foram condrites auricular e nasal, poliartrite não erosiva soronegativa e inflamação ocular. A presença de condrite do trato respiratório superior foi observada durante a evolução clínica.

De maneira geral, não existem diferenças significativas nas manifestações clínicas da PR entre adultos e crianças $^{(1,2,11)}$. O diagnóstico diferencial inclui as doenças do tecido conjuntivo (como a artrite idiopática juvenil [AIJ] e o lúpus eritematoso sistêmico), as vasculites, as doenças auto-inflamatórias (síndromes febris periódicas) e as doenças granulomatosas. Algumas doenças infecciosas com manifestações cutâneas, como a sífilis e a hanseníase, também devem ser consideradas.

Quadro 1

CRITÉrios Diagnósticos PARA POLICONDRITE RECIDIVANTE (MCADAM ET $A L \cdot{ }^{10}$ )

\begin{tabular}{l}
\hline Condrite auricular bilateral \\
Poliartrite não-erosiva inflamatória soronegativa \\
Condrite nasal \\
Inflamação ocular \\
Condrite do trato respiratório \\
Dano audiovestibular \\
\hline
\end{tabular}

Para o diagnóstico são necessários três ou mais critérios.

Embora o nosso paciente tenha apresentado artrite e entesite, lembrando AIJ - artrite relacionada à entesite, a radiografia de sacroilíacas mostrou-se normal, o HLA-B27 foi negativo e não foi evidenciada uveíte aguda. O envolvimento do trato respiratório alto é o principal fator evolutivo de mau prognóstico ${ }^{(1,9)}$. Nosso paciente apresentou acometimento do trato respiratório alto (laringite com estridor respiratório) tardio, um ano após o diagnóstico, e estando o paciente em vigência de terapia imunossupressora. Esta 
temida manifestação indica a necessidade da monitoração da função pulmonar mesmo em pacientes assintomáticos. A mortalidade secundária às complicações respiratórias ocorre entre $10 \%$ e $50 \%$ dos casos $^{(1)}$. Knipp et al. ${ }^{(2)}$ revisaram os dados de literatura de 13 pacientes pediátricos com $\mathrm{PR}$, dos quais $61 \%$ apresentaram acometimento do trato respiratório. Um reconhecimento precoce de queixas respiratórias em um paciente portador de PR requer um tratamento rápido e agressivo com drogas imunossupressoras.

A condrite é uma manifestação precoce em crianças e adultos com PR. Na série de Knipp et al. ${ }^{(2)}$, a condrite nasal foi a manifestação clínica mais encontrada (69\%), seguida pela condrite auricular $(61 \%)$ e pelo envolvimento articular (61\%). Em três grandes estudos envolvendo 337 adultos com PR, a manifestação clínica mais freqüente foi condrite auricular (89\%), seguida por artrite $(72 \%)$ e condrite nasal $(61 \%)^{(9,10,12,13)}$. Inflamação laringotraqueal esteve presente em $55 \%$ dos casos.

As principais manifestações oculares são episclerite ou esclerite, conjuntivite, ceratite ou uveíte presentes em até $60 \%$ dos $\operatorname{casos}^{(9,14)}$. Em nosso paciente, o diagnóstico de conjuntivite foi a primeira manifestação clínica seguido pelos acometimentos articular, nasal e auricular. Knipp el al(2) observaram que $7 / 13$ pacientes apresentaram conjuntivite e/ou perfuração de córnea. Portanto, deve ser realizada avaliação oftalmológica em todos os $\operatorname{casos}^{(2)}$.

Outras manifestações menos freqüentes já foram descritas na literatura e incluem diminuição da audição, disfunção vestibular, valvulopatias mitral e aórtica, vasculite, síndrome mielodisplásica e perda da função renal ${ }^{(9,10,12,13)}$. Até a presente data, nenhuma dessas manifestações foi observada no nosso paciente.

A realização de biópsia de cartilagem para a confirmação diagnóstica não é obrigatória, uma vez que o quadro clínico, na maioria dos casos, é altamente sugestivo da doença. Em alguns casos podem ser observadas complicações decorrentes desse procedimento, como dificuldade de cicatrização e até mesmo infecção secundária. Reina et al. ${ }^{(15)}$ descreveram uma infecção por Pseudomonas aeruginosa após biópsia auricular.

\section{REFERÊNCIAS}

1. Gergely P Jr, Poor G: Relapsing polychondritis. Best Pract Res Clin Rheumatol 18:723-38, 2004.

2. Knipp S, Bier H, Horneff G, et al.: Relapsing polychondritis in childhood - Case report and short review. Rheumatol Int 9:231-4, 2000.
O reduzido número de pacientes com PR e a diversidade das manifestações clínicas são fatores que dificultam a realização de ensaios clínicos randomizados. Portanto, o tratamento da PR é baseado na experiência de especialistas. $\mathrm{O}$ uso de antiinflamatórios não hormonais associado à corticoterapia sistêmica está indicado nos casos de artrite e condrite (nasal e auricular). O uso de imunossupressores como o metotrexato, a azatioprina e a ciclosporina é necessário em muitos casos e tem como principal objetivo o controle do processo inflamatório e o efeito poupador de corticosteróides. A ciclofosfamida por via endovenosa está indicada em casos graves com acometimentos pulmonar, cardíaco, renal e/ou oftalmológico ${ }^{(1,9,14)}$.

Recentemente, alguns pacientes adultos com recidivas freqüentes e refratários à terapia convencional com corticosteróides e imunossupressores foram tratados com agentes biológicos (infliximabe e etanercepte) e apresentaram excelente resposta clínica com diminuição do corticosteróide e, em alguns casos, suspensão dessa classe de medicamentos, além de significativa melhora das provas inflamatórias ${ }^{(3,16-19)}$. Esse fato colabora para o reforço da suspeita da participação das citocinas pró-inflamatórias como mediadores importantes na patogenia da PR.

Vounotrypidis et al.$^{(20)}$ descreveram um caso de um adulto com PR refratária ao uso de vários imunossupressores e de infliximabe, com melhora dos parâmetros inflamatórios após o uso de anakinra (antagonista do receptor da IL-1). Após alguns dias do uso desse biológico, o paciente apresentou melhora importante na proteína C reativa e VHS, sendo suspenso o corticosteróide em um mês. Embora não tenhamos acesso a esse medicamento na rede pública em nosso meio, o anakinra constitui-se numa alternativa terapêutica em casos refratários ao tratamento com imunossupressores.

Em conclusão, destacamos a importância do diagnóstico precoce da $\mathrm{PR}$, uma vez que o quadro clínico é típico e identificável na maioria dos casos na fase inicial da doença. $\mathrm{O}$ tratamento deve ser agressivo, especialmente nos pacientes com manifestações respiratórias. Devemos estar atentos às possíveis recidivas que poderão ocorrer tardiamente.

Declaramos a inexistência de conflitos de interesse.
3. Carter JD: Treatment of relapsing polychondritis with a TNF antagonist. J Rheumatol 32:1413, 2005

4. Yang CL, Brinckmann J, Rui HF, et al.: Autoantibodies to cartilage collagens in relapsing polychondritis. Arch Dermatol Res 285:245-9, 1993.

5. Buckner JH, Van Landeghen M, Kwok WW, Tsarknaridis L: Identification of type II collagen peptide 261-273-specific T 
cell clones in a patient with relapsing polychondritis. Arthritis Rheum 46:238-44, 2002.

6. Lang B, Rothenfusser A, Lanchbury JS, et al.: Susceptibility to relapsing polychondritis is associated with HLA-DR4. Arthritis Rheum 36:660-4, 1993.

7. Taneja V, Griffiths M, Behrens M, Luthra HS, David CS: Auricular chondritis in NOD.DQ8.Abetao (Ag7-/-) transgenic mice resembles human relapsing polychondritis. J Clin Invest 112:1843-50, 2003.

8. Thompson LD: Relapsing polychondritis. Ear Nose Throat J 81(10):705, 2002.

9. Kent PD, Michet CJ Jr, Luthra HS: Relapsing polychondritis. Curr Opin Rheumatol 16:56-61, 2004.

10. McAdam LP, O'Hanlan MA, Bluestone R, Pearson CM: Relapsing polychondritis: prospective study of 23 patients and a review of the literature. Medicine (Baltimore) 55:193-215, 1976.

11. Fonseca AR, Oliveira SK, Sztajnbok FR, Rodrigues MC: Policondrite recidivante - Relato de 3 casos pediátricos. Anais do VI Congresso Brasileiro de Reumatologia Pediátrica, Belo Horizonte, 2006, p. 13.

12. Michet CJ Jr, McKenna CH, Luthra HS, O'Fallon WM: Relapsing polychondritis. Survival and predictive role of early disease manifestations. Ann Intern Med 104:74-8, 1986.
13. Trentham DE, Le CH: Relapsing polychondritis. Ann Intern Med 129:114-22, 1998.

14. Letko E, Zafirakis P, Baltatzis S, Voudouri A, Livir-Rallatos C, Foster CS: Relapsing polychondritis: a clinical review. Semin Arthritis Rheum 31:384-95, 2002.

15. Reina CO, Iriarte MT, Reyes FJ, Monge EG, Barona RL, Angel DG: When is a biopsy justified in a case of relapsing polychondritis? J Laryngol Otol 113(7):663-5, 1999.

16. Saadoun D, Deslandre CJ, Allanore Y, Pham XV, Kahan A: Sustained response to infliximab in 2 patients with refractory relapsing polychondritis. J Rheumatol 30:1394-5, 2003.

17. Richez C, Dumoulin C, Coutouly X, Schaeverbeke T: Successful treatment of relapsing polychondritis with infliximab. Clin Exp Rheumatol 22:629-31, 2004.

18. Mpofu S, Estrach C, Curtis J, Moots RJ: Treatment of respiratory complications in recalcitrant relapsing polychondritis with infliximab. Rheumatology (Oxford) 42:1117-8, 2003.

19. Cazabon S, Over K, Butcher J: The successful use of infliximab in resistant relapsing polychondritis and associated scleritis. Eye 19:222-4, 2005.

20. Vounotrypidis P, Sakellariou GT, Zisopoulos D, Berberidis C: Refractory relapsing polychondritis: rapid and sustained response in the treatment with an IL- 1 receptor antagonist (anakinra). Rheumatology (Oxford) 45:491-2, 2006. 